\title{
Cohort study on the effects of everyday life radio frequency electromagnetic field exposure on non-specific symptoms and tinnitus
}

Patrizia Frei $^{\mathrm{a}, \mathrm{b}}$ Evelyn Mohler $^{\mathrm{a}, \mathrm{b}}$ Charlotte Braun-Fahrländer $^{\mathrm{a}, \mathrm{b}}$ Jürg Fröhlich $^{\mathrm{c}}$ Georg Neubauer $^{\mathrm{d}, \mathrm{e}}$ Martin Röösli ${ }^{\mathrm{a}, \mathrm{b}}$ and the QUALIFEX-team

${ }^{a}$ Swiss Tropical and Public Health Institute, Socinstr. 57, P.O. Box, CH-4002 Basel, Switzerland

${ }^{b}$ University of Basel, Petersplatz 1, CH-4003 Basel, Switzerland

${ }^{c}$ Laboratory for Electromagnetic Fields and Microwave Electronics, ETH Zürich, Gloriastr. 35, CH-8092 Zürich, Switzerland

${ }^{d}$ Seibersdorf Laboratories, EMC \& Optics, A-2444 Seibersdorf, Austria

${ }^{e}$ Austrian Institute of Technology, Safety and Security Department, A-2444 Seibersdorf, Austria

E-mail addresses: Patrizia Frei ${ }^{1}$ : frei@ cancer.dk; Evelyn Mohler: e.mohler@unibas.ch; Charlotte Braun-Fahrländer: c.braun@unibas.ch; Jürg Fröhlich: j.froehlich@ifh.ee.ethz.ch; Georg Neubauer: Georg.Neubauer@ait.ac.at; Martin Röösli: martin.roosli@unibas.ch

\section{Corresponding Author:}

Martin Röösli

Swiss Tropical and Public Health Institute

Socinstrasse 57

P.O. Box

CH-4002 Basel

Phone: +41 (0)61 2848383

Fax: +41 (0)612848101

mailto: martin.roosli@unibas.ch

$\begin{array}{ll}\text { Abbreviations and definitions } \\ \text { RF-EMF } & \text { Radio frequency electromagnetic field } \\ \text { W-LAN } & \text { Wireless local area network } \\ \text { DECT } & \text { Digital enhanced cordless telecommunications } \\ \text { FM } & \text { Frequency modulation } \\ \text { EHS } & \text { Electrohypersensitive }\end{array}$

\footnotetext{
${ }^{1}$ Present address: Institute of Cancer Epidemiology, Danish Cancer Society, Strandboulevarden 49, 2100 Copenhagen $\varnothing$,Denmark
} 


\section{Abstract}

Background: There is public concern regarding potential health effects of radio frequency electromagnetic fields (RF-EMF) exposure, as produced by mobile phones or broadcast transmitters. The objective of this study was to investigate the association between RF-EMF exposure and non-specific symptoms and tinnitus in a prospective cohort study.

Methods: In 2008, 1375 randomly selected participants from Basel, Switzerland, were enrolled in a questionnaire survey with follow-up after one year (participation rate $82 \%$ ). A score for somatic complaints (von Zerssen list) and headache (HIT-6) was assessed. Far-field environmental RF-EMF exposure was predicted using a validated prediction model.

Regarding near-field exposure, self-reported mobile and cordless phone use as well as mobile phone operator data were collected. In multivariate regression models, we investigated whether exposure at baseline (cohort analysis) or changes in exposure between baseline and follow-up (change analysis) were related to changes in health scores.

Results: For participants in the top decile of environmental far-field RF-EMF exposure at baseline, in comparison to participants exposed below the median value, the change in the von Zerssen- and HIT-6-scores between baseline and follow-up was -0.12 (95\%-CI: -1.79 to 1.56) and -0.37 (95\%-CI: -1.80 to 1.07$)$ units, respectively. Exposure to near-field sources and a change in exposure between baseline and follow-up were not related to non-specific symptoms. Similarly, no association between RF-EMF exposure and tinnitus was observed. Conclusions: In this first cohort study using objective and well-validated RF-EMF exposure measures, we did not observe an association between RF-EMF exposure and non-specific symptoms or tinnitus.

Key words: Radio frequency electromagnetic field (RF-EMF), exposure assessment, mobile phone base station, Wireless LAN, DECT cordless phone, radio / television broadcast 


\section{Introduction}

Radio frequency electromagnetic field (RF-EMF) emitting sources like mobile phone base stations and handsets or broadcast transmitters are ubiquitous and exposure has been increasing over the past 20 years (Neubauer et al. 2007). This development has raised public concerns regarding potentially detrimental health effects of this technology, especially regarding effects on non-specific symptoms like headache (Blettner et al. 2009; Röösli et al. 2004; Schreier et al. 2006; Schröttner and Leitgeb 2008).

Several studies have addressed potential effects of RF-EMF exposure on non-specific symptoms so far. Most studies were performed in laboratories, e.g. (Cinel et al. 2008; Hillert et al. 2008; Regel et al. 2007). The advantage of laboratory trials is that the well-defined exposure setting allows for the exact determination of a person's exposure level as well as randomization and double-blinding. The disadvantages are that usually only a small study population can be investigated and that effects after prolonged exposure durations cannot be studied due to ethical and practical reasons. Such effects can only be addressed in epidemiological studies. However, sound assessment of RF-EMF exposure in everyday life is highly challenging (ICNIRP 2009). The use of crude exposure proxies, like the lateral distance to the closest mobile phone base station, has been shown to be inappropriate (Bornkessel et al. 2007; Frei et al. 2010; Neubauer et al. 2007; Schüz and Mann 2000). More sophisticated exposure assessment methods such as spot or personal measurements need considerable efforts and thus, most epidemiological studies conducted so far were of crosssectional design (Balikci et al. 2005; Berg-Beckhoff et al. 2009; Blettner et al. 2009; Chia et al. 2000; Heinrich et al., 2011; Mohler et al. 2010; Thomas et al. 2008), which are restricted in terms of drawing conclusions about a causal relationship between exposure and health (Seitz et al. 2005). In addition, spurious exposure-outcome associations can be introduced if there is information bias or a nocebo effect, i.e. the development of symptoms due to 
concerns, evidence for which has been provided by several laboratory trials (Röösli et al. 2010a; Rubin et al. 2010). In cross-sectional studies even inverse associations between exposure and health may be observed, if persons claiming to be electrohypersensitive (EHS), i.e. to develop symptoms due to RF-EMF exposure, avoid RF-EMF exposure, since such individuals usually suffer more often from non-specific symptoms than the general population (Landgrebe et al. 2009; Seitz et al. 2005).

In the framework of the QUALIFEX study (health related quality of life and radio frequency electromagnetic field exposure: prospective cohort study), we performed a baseline questionnaire survey in 2008 in a random population sample. One year later, a follow-up was conducted. Due to the unknown biological mechanism of RF-EMFs below the thermal threshold, we included several exposure surrogates for both environmental far-field sources (e.g. mobile phone base stations) as well as near-field sources (e.g. mobile phones). The aim of this study was to investigate whether RF-EMF exposure at baseline or a change of RFEMF exposure between baseline and follow-up was associated with the development of nonspecific symptoms of ill health or tinnitus.

\section{Methods}

\subsection{Study population}

The recruitment strategy of the baseline survey is described in detail in Mohler et al. ( 2010). In brief, in May 2008 we sent out questionnaires entitled "environment and health" to 4000 randomly selected residents from the region of Basel, Switzerland, aged between 30 and 60 years. After one year, a follow-up was conducted by sending the same questionnaire to the respondents of the baseline survey. Reasons for non-eligibility at both surveys were severe disabilities, death, incorrect addresses, absence during the time of the survey or language problems. 


\subsection{Written questionnaire: health and exposure assessment}

The written questionnaire consisted of three parts. The first part contained questions related to the health status of the previous 4 months of each investigation. The study participants were asked to fill in several standardized questions, namely the 24-item list of somatic complaints (von Zerssen, e.g. fatigue, loss of appetite, lack of energy or concentration) (von Zerssen 1976) and the six-item headache impact test (HIT-6) (Kosinski et al. 2003). The von Zerssen-score ranges from 0 (no complaints) to 72 (severe complaints), and the HIT-6-score from 36 (no impact) to 78 (severe impact). In addition, the participants were asked to report whether they suffered from tinnitus at the time of the survey.

In the second part of the questionnaire, we assessed RF-EMF exposure. Exposure to environmental far-field sources as well as to near-field sources were considered. Regarding exposure to environmental far-field sources, we used two surrogates:

1) Residential exposure to fixed site transmitters (mobile phone base stations and broadcast transmitters), calculated by means of a geospatial propagation model which had been developed and validated for the study region (Bürgi et al. 2008; Bürgi et al. 2010).

2) Total personal exposure, assessed using a predictive exposure assessment model (considered sources: broadcast transmitters, mobile phone handsets and base stations, digital enhanced cordless telecommunications (DECT) phones and wireless LAN). This model was developed and validated based on personal exposure measurements in an independent study sample of 166 residents from the same study region and is described in Frei et al. (2009). The model includes the following exposure relevant characteristics: residential RF-EMF exposure predicted with the geospatial propagation model, modified by the type of house wall and type of window frames, ownership of wireless communication devices (W-LAN, mobile and cordless phones) and behavioral characteristics (amount of time spent in public transport vehicles or cars, percent full-time equivalent). 
Regarding near-field exposure, three exposure surrogates were used:

1) Self-reported mobile phone use (using 7 categories from "never" to "more than 5 times a day (including an estimate of the duration of use per day in the relevant categories)

2) Self-reported cordless phone use (using 7 categories from "never" to "more than 60 minutes per day")

3) Network operator data on all in- and outgoing private mobile phone calls of the previous 6 months of each investigation for participants who gave written informed consent.

Finally, we asked participants to compare their personal exposure situation with the average Swiss population. An association between perceived exposure and health, independent of actual exposure, would be indicative of nocebo effects or information bias.

The third part of the questionnaire contained questions on socio-demographic factors (e.g. age, gender). We also asked the participants whether they were electrohypersensitive (EHS) (defined as answering "yes" to either the question “Are you electrohypersensitive?" or to the question "Do you think that you develop detrimental health symptoms due to electromagnetic pollution in everyday life?”).

\subsection{Statistical analyses}

For all analyses, the linear outcome variables (von Zerssen- and HIT-6-score) were analyzed using linear regression models and the binary tinnitus variable was analyzed using logistic regression models. For each outcome, cross-sectional analyses were performed for the baseline and follow-up survey. In addition, we performed a cohort analysis and a change analysis. For the cohort analysis, we assessed the association between the exposure level at baseline and the change in health status between baseline and follow-up. The cross-sectional and cohort analyses were based on three exposure categories: exposure below median (reference), $50^{\text {th }}$ to $90^{\text {th }}$ percentile and the top exposure decile. In the change analysis, we examined whether the change in exposure between baseline and follow-up resulted in a 
change in health outcome. We compared the study participants with the $20 \%$ largest decrease and increase with the remaining $60 \%$ who experienced a smaller or no change of exposure between baseline and follow-up (reference).

All models were adjusted for age, sex, body mass index, stress, physical activity, smoking habits, alcohol consumption, education, marital status, degree of urbanity, nightshift work, belief in health effects due to RF-EMF exposure, use of sleeping drugs and general attitude towards the environment. In the cohort and change analyses, we adjusted for confounders at baseline and additionally adjusted the models for moving house between the two surveys. Missing values in the confounder variables (between 0 and 5.5\%) at baseline were replaced with the information of the follow-up and vice versa. If missing at both surveys (between 0 and $1.1 \%$ for the variables included in the models), the most common category (categorical variables) or the mean value (linear variables) was used. (Self-reported) use of mobile and cordless phones were included as co-exposures in all models for far-field exposure sources, and total personal far-field exposure was used as co-exposure variable in all models for mobile and cordless phone use. In the model for self-estimated exposure, personal total exposure and cordless and mobile phone use were included.

All models were tested for interaction between EHS status and the exposure measures. The presented coefficients and odds ratios (ORs) therefore represent estimates for the nonEHS individuals. The interaction term was tested with likelihood-ratio tests. An additional sensitivity analysis was conducted including an interaction term for age (30-44 vs. 45-60 years) and the different exposure metrics to investigate whether the RF-EMF effect differed for the two age groups. Statistical analyses were carried out using STATA version 10.1 (StataCorp, College Station, TX, USA). Ethical approval for the conduct of the study was received from the ethical committee of Basel on March 19, 2007 (EK: 38/07). 


\section{Results}

\subsection{Study participants}

Response rate was $37 \%$ at baseline and $82 \%$ at follow-up (Fig. 1). The characteristics of the study participants are listed in Table 1. In general, there were only small differences between study participants who participated at follow-up compared with participants of the baseline survey. The mean age was 46 years (standard deviation (sd): 9 years) at baseline and 47 years (sd: 9 years) one year later at follow-up.

\subsection{RF-EMF exposure}

Table 2 shows the ranges of the RF-EMF levels in all exposure categories of the various exposure metrics at baseline and follow-up as well as the changes between baseline and follow-up. Mean total personal far-field exposure was $0.12 \mathrm{~mW} / \mathrm{m}^{2}(0.21 \mathrm{~V} / \mathrm{m})$ at baseline and $0.13 \mathrm{~mW} / \mathrm{m}^{2}(0.22 \mathrm{~V} / \mathrm{m})$ at follow-up. Mean residential exposure to fixed site transmitters was $0.02 \mathrm{~mW} / \mathrm{m}^{2}(0.09 \mathrm{~V} / \mathrm{m})$ at baseline and follow-up. The study participants reported to use their mobile phones at baseline and at follow-up for a mean of 1.18 hours and 1.13 hours per week, respectively, and their cordless phones for 1.26 hours and 1.28 hours per week, respectively. Persons for whom operator data were available used their mobile phone on average during 31 minutes per week at baseline $(n=539)$ and during 21 minutes per week at follow-up ( $\mathrm{n}=424)$. The self-reported use of the private mobile phone restricted to the persons providing operator data was 28 minutes at baseline and 30 minutes at follow-up.

\subsection{Association between RF-EMF exposure and health outcomes}

At baseline, the average von Zerssen-score for somatic complaints was 12, ranging from 0 to 57. At follow up, it was 13, ranging from 0 to 66 . The average HIT-6-score (headache) was 46 at baseline (range: 36-78) and at follow-up (range: 36-74). 128 (9\%) 
persons reported to suffer from tinnitus at baseline and $131(12 \%)$ at follow-up. Twenty persons reported to suffer from tinnitus only at baseline and 44 only at follow-up.

Figures A.1-3 show the results of the cross-sectional analyses and Fig. 2-4 the results of the cohort and change analyses. Of the 144 computed risk estimates (96 regression coefficients and 48 odds ratios), 7 (5\%) estimates reached statistical significance. The von Zerssen-score dropped by -1.79 units (95\% confidence interval (CI): -3.56 to -0.02$)$ and by 1.42 (95\%-CI: -2.67 to -0.17) between baseline and follow-up for individuals in the top decile of self-reported mobile phone use and for individuals who increased their mobile phone use from baseline to follow-up, respectively (Fig. 2). Participants in the middle exposure group $\left(50^{\text {th }}\right.$ to $90^{\text {th }}$ percentile) of self-reported mobile phone use at follow-up had a lower von Zerssen-score (Fig. A.1, cross-sectional analysis follow-up, -1.66 points, 95\%-CI: -2.96 to 0.35). This trend for an inverse association between self-reported mobile phone use and somatic complaints was quite consistent in all analyses, however not confirmed with network operator data. Participants who believed to be more exposed than the general Swiss population at baseline were more likely to have an increased von Zerssen-score at follow-up (Fig. 2, cohort analysis, +2.27 points, $95 \%$-CI: 0.05 to 4.49 ). Such a tendency could also be seen in the cross-sectional data. A similar trend could be observed in the HIT-6 headache score, where the score increased more in individuals who rated their self-estimated exposure higher at follow-up compared to baseline (Fig. 3, change analysis, +1.21 points, $95 \%$-CI: 0.14 to 2.29). Participants in the middle exposure category for residential exposure to fixed site transmitters had a statistically significantly increased headache score in the cohort analysis (Fig. $3,+0.91$ points, $95 \%$-CI: 0.07 to 1.75$)$ and a decreased headache score (-1.23 points, 95\%-CI: -2.22 to -0.24 ) in the baseline survey (Fig. A.2). No statistically significant association between RF-EMF exposure and tinnitus was found (Figs. 4 and A.3). There was no consistent difference between EHS and non-EHS individuals regarding the exposureoutcome associations based on the interaction term between EHS status and exposure (data 
not shown). In addition, the sensitivity analyses did not provide evidence for any outcome that the effect was different for younger and older study participants (30-44 and 45-60 years) (data not shown).

\section{Discussion}

Our findings do not suggest an association between far- or near-field RF-EMF exposure in everyday life and the development of non-specific symptoms or tinnitus. We observed 7 statistically significant associations out of 144 risk estimates, which is to be expected by chance alone. Furthermore, the observed associations did not show a consistent pattern.

\subsection{Strengths and limitations}

The longitudinal study design allows for more robust conclusions compared to previous cross-sectional studies (e.g. Balikci et al. 2005; Berg-Beckhoff et al. 2009; Heinrich et al., 2011, Thomas et al. 2008). The subjective symptoms (von Zerssen and HIT-6) were self-reported, but based on standardized questions. To our knowledge, our study used the most comprehensive exposure assessment method by taking into account potential effects of both exposure to environmental far-field and near-field sources. For both exposure types, we used objective exposure data. The elaborate predictive exposure assessment model includes all relevant RF-EMF exposure sources in everyday life in the frequency range of 88-2500 MHz. It is based on the geospatial propagation model that includes very accurate parameters from all fixed site transmitters of the study region, complemented with data on relevant behaviors. The feasibility and reproducibility of the prediction model as well as of the geospatial propagation model was previously demonstrated (Bürgi et al. 2010; Frei et al. 2009). $39.2 \%$ of the study participants at baseline and of $37.8 \%$ at follow-up provided objective traffic records of the previous 6 months of each investigation, which has to our 
knowledge not been done in previous studies investigating the effect of mobile phone use on non-specific symptoms. Unfortunately, we were only able to obtain traffic records of private, but not business mobile phones. About $25 \%$ of the individuals who agreed to provide their traffic records at baseline and follow-up owned a business mobile phone as well. This may have led to some exposure misclassification.

Another limitation was the rather low participation rate of $37 \%$ in the baseline survey. If participation was related to both, health and exposure status, selection bias is of concern. There is no evidence in the data that persons suffering from more symptoms were substantially more likely to participate in the baseline survey, since we found a similar HIT-6score and even slightly lower von Zerssen-score (less complaints) in comparison to a recent German study, where persons were selected from a nationwide survey and the participation rate was very high (85\%) (Berg-Beckhoff et al. 2009). In the follow-up, participation rate was high $(82 \%)$ and health scores were similar as compared to the baseline survey. The latter might indicate that healthier individuals were slightly more likely to participate in the followup if one assumes a decrease of health status with age. Regarding far-field exposure, we observed no evidence for a difference between participants and non-participant of the followup (total personal exposure: $0.120 \mathrm{~mW} / \mathrm{m}^{2}$ vs. $0.122 \mathrm{~mW} / \mathrm{m}^{2}$ ) and thus the risk estimates for far-field RF-EMF are not expected to be biased. However, mobile phone use at baseline was higher for individuals not participating compared to individuals who participated at follow-up (self-reported: 90 vs. 63 minutes/week and operator data: 43 vs. 28 minutes/week). In combination with a potential selection of more healthier individuals, this might explain the few protective effects found for mobile phone use, although the extent of bias cannot be large given the high participation rate at follow-up. Similarly, there was some indication that risk estimates of cross-sectional analyses for mobile and cordless phone use may be biased downwards based on a non-responder analysis with 654 individuals who did not participate at the baseline survey but who answered a short telephone interview (Mohler et al., 2010). An 
alternative explanation for these protective risks found for mobile and cordless phone use might also be reverse causality, meaning that healthier individuals use their mobile phone more often compared to less healthy individuals.

\subsection{Interpretation}

In general, we found no evidence that exposure to RF-EMF in everyday life is associated with the development of non-specific symptoms or tinnitus. The few statistically significant effects were not consistent. A total of $5 \%$ of the tests were statistically significant, which can be expected by chance. We conducted a large number of analyses because in the absence of a known biological mechanism in the low dose range, it was unclear which aspect of exposure might be relevant for health disturbances, if any at all. We did not apply a formal multiple endpoint correction (e.g. Bonferroni correction). Instead we checked the consistency and biological plausibility of similar analyses. The statistical power of the study was adequate to detect relatively small changes of the health outcome: a post-hoc power analysis revealed that a change of 1.6 points in the von Zerssen-score and of 1.4 points in the HIT-6-score for the highest exposure decile could have been detected with a power of $80 \%$. To compare, the von Zerssen and HIT-6-score of persons who felt disturbed by noise of their neighbors were higher by 5.1 and 2.8 points, respectively, in comparison to persons who did not feel disturbed.

With regard to environmental far-field sources, our findings are in line with laboratory trials investigating acute effects of whole-body mobile phone base station exposure (Röösli et al. 2010a). In epidemiological studies, there is a tendency that effects are found in studies where crude or subjective exposure surrogates were used, while for studies using objective exposure surrogates mostly no effect was found (Röösli et al. 2010a). A similar tendency was also noticeable in our study. Self-estimated exposure was significantly associated with symptom scores in two out of eight analyses, and most of the non-significant associations 
showed a similar trend. This indicates that nocebo effect and/or information bias may play a role when using self-estimated exposure measures. This is in line with experimental studies (Röösli et al. 2010a; Rubin et al. 2010).

Regarding near-field sources, no acute effects of mobile phone-like exposure were observed in laboratory trials (Nam et al. 2009; Röösli 2008; Stovner et al. 2008) except for one study, where a higher headache score was found after applying a $3 \mathrm{~h}$ mobile phone-like exposure (Hillert et al. 2008). In some of the earlier cross-sectional epidemiological studies, positive associations were found (Balikci et al. 2005; Chia et al. 2000). Selection bias, information bias and nocebo, might have played an important role in previous epidemiological studies, but are of less concern in our cohort and change analyses except for a possible selection bias regarding mobile phone use discussed above. Another reason for this discrepancy might be that the participants in our study were more accurate in reporting their mobile phone use because they were aware that we collected the data from their mobile phone operators as well, which might have reduced information bias.

We did not find an association between RF-EMF exposure and the development of tinnitus. This is in line with previous research (Davidson and Lutman 2007; Mortazavi et al. 2007; Thomas et al. 2008), except for one Austrian study (Hutter et al. 2010), where increased risks for tinnitus were found for individuals who used their mobile phone for at least 4 years. In that study, however, self-reported data on the use of mobile phones was used, which is of concern in case-control studies (Vrijheid et al. 2006).

We found no indication that EMF exposure is more harmful for EHS individuals, which is in line with laboratory trials. A more detailed analysis of the characteristics and exposure effects in the EHS collective of this survey is given in Röösli et al. (2010b).

Generally, the mean exposure levels to environmental far-field RF-EMF sources in our study population were by several orders of magnitude below the current standard limits. We observed only small individual exposure differences between baseline and follow-up, and our 
data do not allow us to investigate longer-term effects after several years of exposure. In addition, our results apply only to adults in the age range of 30-60 years. We can therefore only state that effects in adults aged 30-60 years due to these small exposures and exposure changes in a time window of one year are unlikely. However, we cannot draw conclusions about health effects which might occur due to higher exposure changes at levels close to the standard limits, after longer-term induction periods, or about effects in children or the elderly.

To conclude, we did not find evidence for a detrimental effect of exposure to RF-EMF in everyday life on the development of non-specific symptoms or tinnitus. These results, however, are only valid for relatively small levels of RF-EMF exposure that occur today. We cannot make firm conclusions about higher exposure levels or more dramatic changes of exposure that might be induced by the future technical development.

\section{Acknowledgements}

We thank Matthias Egger, Niklas Joos, Axel Hettich (QUALIFEX team) for inspiring discussions. We thank Fabian Trees from the Swiss Federal Statistical Office for providing the geographical coordinates of the study participants and the statistical department of Basel for providing the addresses of the study participants. Many thanks go also to all study participants who volunteered for the study.

\section{Funding}

The study was funded by the Swiss National Science Foundation (Grant 405740-113595). Martin Röösli is supported by the Swiss School of Public Health + (SSPH+).

\section{Conflict of interest}

The authors declare no conflict of interest. 


\section{References}

Balikci K, Cem Ozcan I, Turgut-Balik D, Balik HH. 2005. A survey study on some neurological symptoms and sensations experienced by long term users of mobile phones. Pathol Biol (Paris) 53:30-34.

Berg-Beckhoff G, Blettner M, Kowall B, Breckenkamp J, Schlehofer B, Schmiedel S et al. 2009. Mobile phone base stations and adverse health effects: phase 2 of a crosssectional study with measured radio frequency electromagnetic fields. Occupational and environmental medicine 66:124-130.

Blettner M, Schlehofer B, Breckenkamp J, Kowall B, Schmiedel S, Reis U et al. 2009. Mobile phone base stations and adverse health effects: phase 1 of a population-based, crosssectional study in Germany. Occupational and environmental medicine 66:118-123.

Bornkessel C, Schubert M, Wuschek M, Schmidt P. 2007. Determination of the general public exposure around GSM and UMTS base stations. Radiat Prot Dosimetry 124:4047.

Bürgi A, Frei P, Theis G, Mohler E, Braun-Fahrländer C, Fröhlich J et al. 2010. A model for radiofrequency electromagnetic fields at outdoor and indoor locations for use in an epidemiological study. Bioelectromagnetics 31:226-236.

Bürgi A, Theis G, Siegenthaler A, Röösli M. 2008. Exposure modeling of high-frequency electromagnetic fields. J Expo Sci Environ Epidemiol 18:183-191.

Chia SE, Chia HP, Tan JS. 2000. Prevalence of headache among handheld cellular telephone users in Singapore: a community study. Environ Health Perspect 108:1059-1062. 
Cinel C, Russo R, Boldini A, Fox E. 2008. Exposure to mobile phone electromagnetic fields and subjective symptoms: a double-blind study. Psychosom Med 70:345-348.

Davidson HC, Lutman ME. 2007. Survey of mobile phone use and their chronic effects on the hearing of a student population. Int J Audiol 46:113-118.

Frei P, Mohler E, Bürgi A, Fröhlich J, Neubauer G, Braun-Fahrländer C et al. 2009. A prediction model for personal radio frequency electromagnetic field exposure. Sci Total Environ 408:102-108.

Frei P, Mohler E, Bürgi A, Fröhlich J, Neubauer G, Braun-Fahrländer C et al. 2010. Classification of personal exposure to radio frequency electromagnetic fields (RFEMF) for epidemiological research: Evaluation of different exposure assessment methods. Environ Int 36:714-720.

Heinrich S, Thomas S, Praml G, von Kries R, Radon K 2011. The impact of exposure to radio frequency electromagnetic fields on chronic well-being in young people - a crosssectional study based on personal dosimetry. Environ Int 37: 26-30.

Hillert L, Akerstedt T, Lowden A, Wiholm C, Kuster N, Ebert S et al. 2008. The effects of $884 \mathrm{MHz}$ GSM wireless communication signals on headache and other symptoms: an experimental provocation study. Bioelectromagnetics 29:185-196.

Hutter HP, Moshammer H, Wallner P, Cartellieri M, Denk-Linnert DM, Katzinger M et al. 2010. Tinnitus and mobile phone use. Occup Environ Med 67: 804-808.

ICNIRP. 2009. Exposure to high frequency electromagnetic fields, biological effects and health consequences $(100 \mathrm{kHz}-300 \mathrm{GHz})$. International Commission on Non-Ionizing Radiation Protection Available: http://www.icnirp.org/documents/RFReview.pdf (accessed 15 July 2009). 
Kosinski M, Bayliss MS, Bjorner JB, Ware JE, Jr., Garber WH, Batenhorst A et al. 2003. A six-item short-form survey for measuring headache impact: the HIT-6. Qual Life Res 12:963-974.

Landgrebe M, Frick U, Hauser S, Hajak G, Langguth B. 2009. Association of tinnitus and electromagnetic hypersensitivity: hints for a shared pathophysiology? PLoS One 4:e5026.

Mohler E, Frei P, Braun-Fahrländer C, Fröhlich J, Neubauer G, Röösli M. 2010. Effects of everyday radiofrequency electromagnetic-field exposure on sleep quality: a crosssectional study. Radiat Res 174:347-356.

Mortazavi SM, Ahmadi J, Shariati M. 2007. Prevalence of subjective poor health symptoms associated with exposure to electromagnetic fields among university students. Bioelectromagnetics 28:326-330.

Nam KC, Lee JH, Noh HW, Cha EJ, Kim NH, Kim DW. 2009. Hypersensitivity to RF fields emitted from CDMA cellular phones: A provocation study. Bioelectromagnetics 30:641-650.

Neubauer G, Feychting M, Hamnerius Y, Kheifets L, Kuster N, Ruiz I et al. 2007. Feasibility of future epidemiological studies on possible health effects of mobile phone base stations. Bioelectromagnetics 28:224-230.

Regel SJ, Tinguely G, Schuderer J, Adam M, Kuster N, Landolt HP et al. 2007. Pulsed radiofrequency electromagnetic fields: dose-dependent effects on sleep, the sleep EEG and cognitive performance. Journal of sleep research 16:253-258.

Röösli M. 2008. Radiofrequency electromagnetic field exposure and non-specific symptoms of ill health: a systematic review. Environ Res 107:277-287. 
Röösli M, Frei P, Mohler E, Hug K. 2010a. Systematic review on the health effects of radiofrequency electromagnetic field exposure from mobile phone base stations. Bull World Health Organ 88:887-896.

Röösli M, Mohler E, Frei P. 2010b. Sense and sensibility in the context of radiofrequency electromagnetic field exposure. Comptes Rendus Physique 11:576-584.

Röösli M, Moser M, Baldinini Y, Meier M, Braun-Fahrländer C. 2004. Symptoms of ill health ascribed to electromagnetic field exposure--a questionnaire survey. Int J Hyg Environ Health 207:141-150.

Rubin GJ, Nieto-Hernandez R, Wessely S. 2010. Idiopathic environmental intolerance attributed to electromagnetic fields (formerly 'electromagnetic hypersensitivity'): An updated systematic review of provocation studies. Bioelectromagnetics 31:1-11.

Schreier N, Huss A, Röösli M. 2006. The prevalence of symptoms attributed to electromagnetic field exposure: a cross-sectional representative survey in Switzerland. Soz Präventivmed 51:202-209.

Schröttner J, Leitgeb N. 2008. Sensitivity to electricity--temporal changes in Austria. BMC Public Health 8:310.

Schüz J, Mann S. 2000. A discussion of potential exposure metrics for use in epidemiological studies on human exposure to radiowaves from mobile phone base stations. J Expo Anal Environ Epidemiol 10:600-605.

Seitz H, Stinner D, Eikmann T, Herr C, Röösli M. 2005. Electromagnetic hypersensitivity (EHS) and subjective health complaints associated with electromagnetic fields of mobile phone communication--a literature review published between 2000 and 2004 . Sci Total Environ 349:45-55. 
Stovner LJ, Oftedal G, Straume A, Johnsson A. 2008. Nocebo as headache trigger: evidence from a sham-controlled provocation study with RF fields. Acta Neurol Scand Suppl 188:67-71.

Thomas S, Kühnlein A, Heinrich S, Praml G, Nowak D, von Kries R et al. 2008. Personal exposure to mobile phone frequencies and well-being in adults: a cross-sectional study based on dosimetry. Bioelectromagnetics 29:463-470.

von Zerssen D. 1976. Complaint list. Manual. Weinheim: Beltz.

Vrijheid M, Deltour I, Krewski D, Sanchez M, Cardis E. 2006. The effects of recall errors and of selection bias in epidemiologic studies of mobile phone use and cancer risk. J Expo Sci Environ Epidemiol 16:371-384. 


\section{Table captions}

Table 1: Characteristics of study participants at baseline and follow-up

Table 2: Exposure levels to different exposure sources at baseline and follow-up and change between baseline and follow-up.

\section{Figure captions}

Fig. 1: Schematic illustration of the study design and the response rates of the baseline and follow-up surveys.

Fig. 2: Results of the cohort analysis and change analysis showing the association between the different exposure surrogates and the change in the von Zerssen-score (linear regression analyses with $95 \%$ confidence intervals (CI)). Negative coefficients indicate an inverse association and positive coefficients a positive association between exposure and somatic complaints.

Fig. 3: Results of the cohort analysis and change analysis showing the association between the different exposure surrogates and the change in the HIT-6 score (linear regression analyses with $95 \%$ confidence intervals (CI)). Negative coefficients indicate an inverse association and positive coefficients a positive association between exposure and headache.

Fig. 4 Results of the cohort analysis and change analysis showing the association between the different exposure surrogates and the development of tinnitus (logistic regression analyses, 
odds ratios (ORs) with 95\% confidence intervals (CI)). ORs $<1$ indicate an inverse and $>1$ a positive association between exposure and tinnitus. 


\section{Appendix A: Web figures}

Fig. A. 1: Results of the cohort two cross-sectional analyses (baseline and follow-up) showing the association between the different exposure surrogates and the von Zerssen-score (regression coefficient with 95\% confidence intervals (CI)). Negative coefficients indicate an inverse association and positive coefficients a positive association between exposure and somatic complaints.

Fig. A. 2: Results of the cohort two cross-sectional analyses (baseline and follow-up) showing the association between the different exposure surrogates and the HIT-6-score (regression coefficient with $95 \%$ confidence intervals (CI)). Negative coefficients indicate an inverse association and positive coefficients a positive association between exposure and somatic complaints.

Fig. A. 3: Results of the cohort two cross-sectional analyses (baseline and follow-up) showing the association between the different exposure surrogates and tinnitus (odds ratios (ORs) with $95 \%$ confidence intervals $(\mathrm{CI}))$. ORs $<1$ indicate an inverse and $>1$ a positive association between exposure and tinnitus. 
Table 1: Characteristics of study participants at baseline and follow-up

\begin{tabular}{|c|c|c|c|c|}
\hline & $\begin{array}{c}\text { Baseline } \\
\text { survey } \\
(n=1375)\end{array}$ & $\%$ & $\begin{array}{l}\text { Follow-up } \\
\text { survey } \\
(n=1122)^{a}\end{array}$ & $\%$ \\
\hline \multicolumn{5}{|l|}{ Age (years) } \\
\hline$<41$ & 407 & 29.6 & 297 & 26.5 \\
\hline $41-50$ & 490 & 35.6 & 357 & 31.8 \\
\hline$>51$ & 478 & 34.8 & 468 & 41.7 \\
\hline \multicolumn{5}{|l|}{ Sex } \\
\hline Female & 798 & 58.0 & 678 & 60.4 \\
\hline Male & 577 & 42.0 & 444 & 39.6 \\
\hline \multicolumn{5}{|l|}{ Health status } \\
\hline (Very) good & 1223 & 89.7 & 983 & 88.6 \\
\hline Half-half & 122 & 8.9 & 112 & 10.1 \\
\hline (Very) bad & 19 & 1.4 & 14 & 1.3 \\
\hline \multicolumn{5}{|l|}{ Educational level } \\
\hline None & 89 & 6.6 & 57 & 5.2 \\
\hline Apprenticeship & 663 & 48.5 & 523 & 47.8 \\
\hline Higher education & 615 & 45.0 & 515 & 47.0 \\
\hline \multicolumn{5}{|c|}{ Belief in health effects due to RF-EMF exposure ${ }^{b}$} \\
\hline No & 82 & 6.0 & 53 & 4.7 \\
\hline Yes & 1069 & 77.7 & 874 & 77.9 \\
\hline Don't know/missing & 224 & 16.3 & 195 & 17.4 \\
\hline \multicolumn{5}{|c|}{ Electromagnetic hypersensitivity $^{c}$} \\
\hline No & 825 & 60.0 & 642 & 57.2 \\
\hline Yes & 294 & 21.4 & 247 & 22.0 \\
\hline Don't know/missing & 256 & 18.6 & 233 & 20.8 \\
\hline \multicolumn{5}{|c|}{ Self-estimated RF-EMF exposure ${ }^{d}$} \\
\hline Lower & 403 & 29.3 & 397 & 35.4 \\
\hline Equal & 576 & 41.9 & 492 & 43.9 \\
\hline Higher & 105 & 7.6 & 69 & 6.1 \\
\hline Don't know/missing & 291 & 21.2 & 164 & 14.6 \\
\hline
\end{tabular}

${ }^{\mathrm{a}}$ Two responders of the follow-up were excluded from the analyses because they went abroad after the baseline survey.

${ }^{\text {b }}$ Question: "Do you believe that there are persons who develop adverse health effects due to electromagnetic pollution in the everyday environment"

'Defined as answering "yes" to one or both of the questions "Are you electrohypersensitive?" and "Do you think that you develop detrimental health symptoms due to electromagnetic pollution in everyday life?" 
${ }^{\text {d}}$ Question: "How would you estimate your personal exposure to the following sources in comparison with the average Swiss population: broadcast transmitters, mobile phone base stations, mobile phones, cordless phones, W-LAN?““ 
Table 1: Exposure levels to different exposure sources at baseline and follow-up and change between baseline and follow-up.

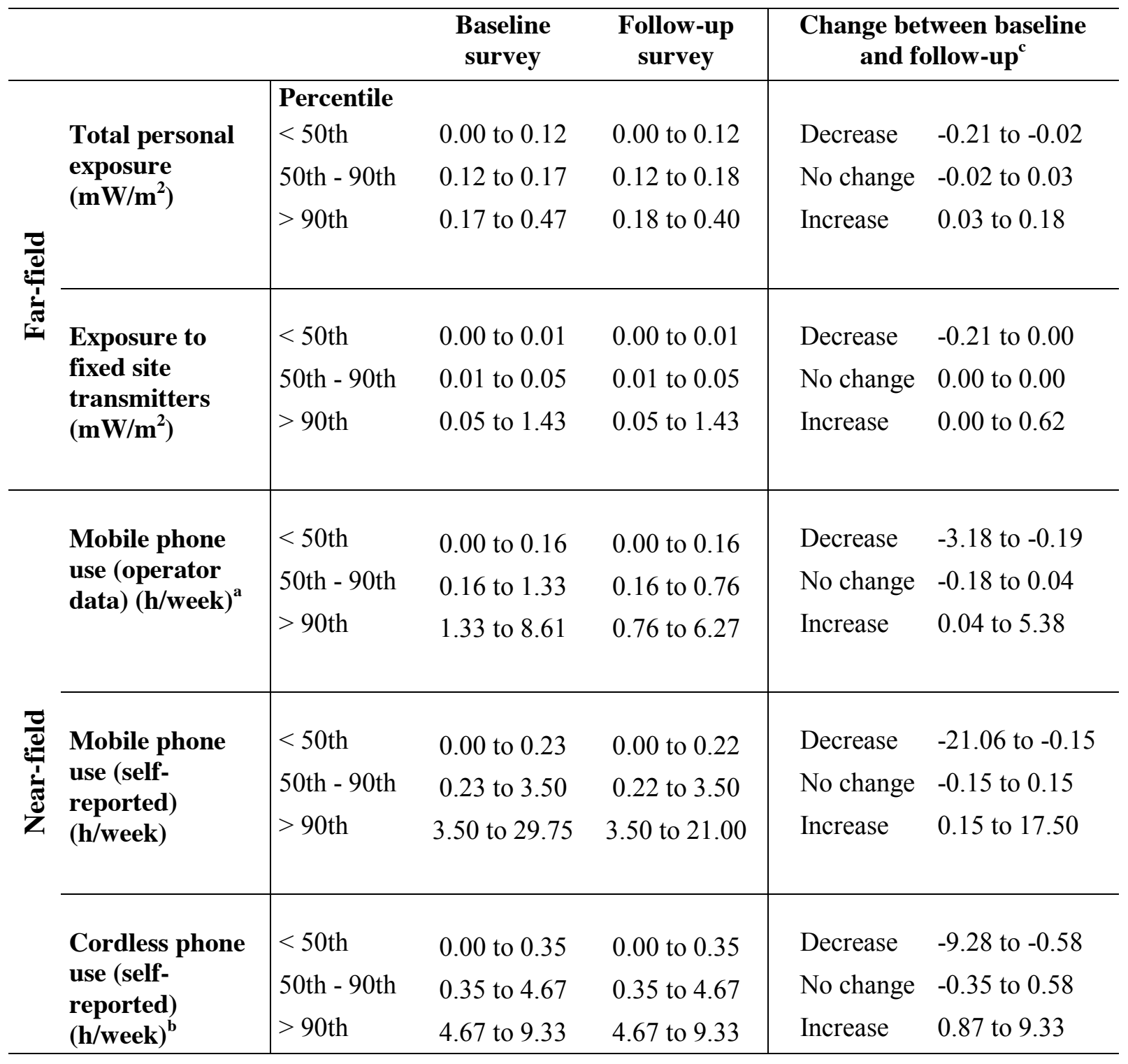

${ }_{n}^{a}=539 / 424$ at baseline/follow-up

${ }^{\mathrm{b}}$ similar values due to the use of categories in the questionnaire

${ }^{c}$ Decrease/increase: study participants with the $20 \%$ largest decrease or increase, no change: smaller or no change. 
Fig. 1

Baseline survey

Postal questionnaire 1 4000 randomly selected persons

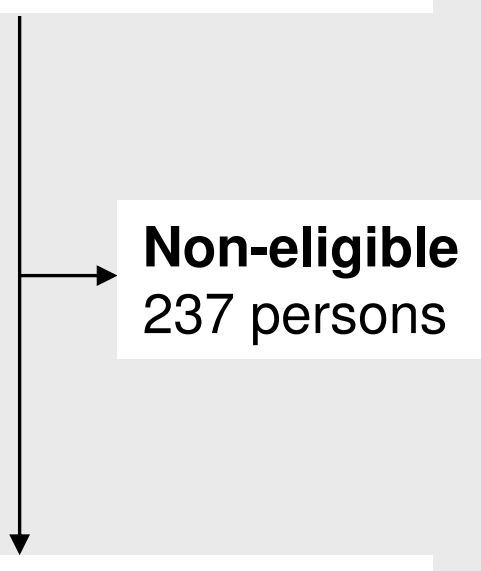

\section{Responders}

1375 study participants (response rate: $37 \%$ )
Follow-up survey

Postal questionnaire 2

1375 participants from the baseline survey

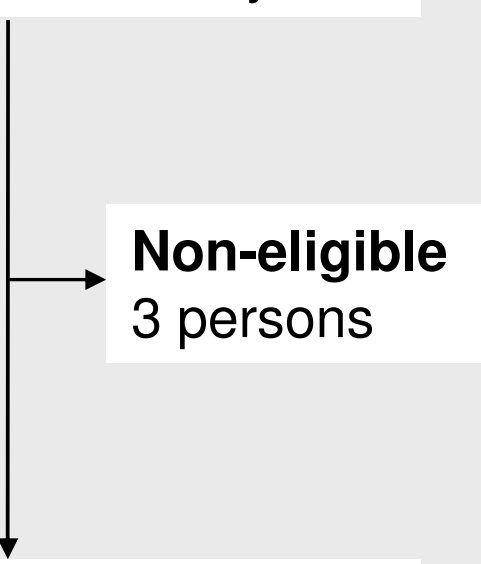

\section{Responders}

1124 study participants (response rate: $82 \%$ ) 
Figure 2

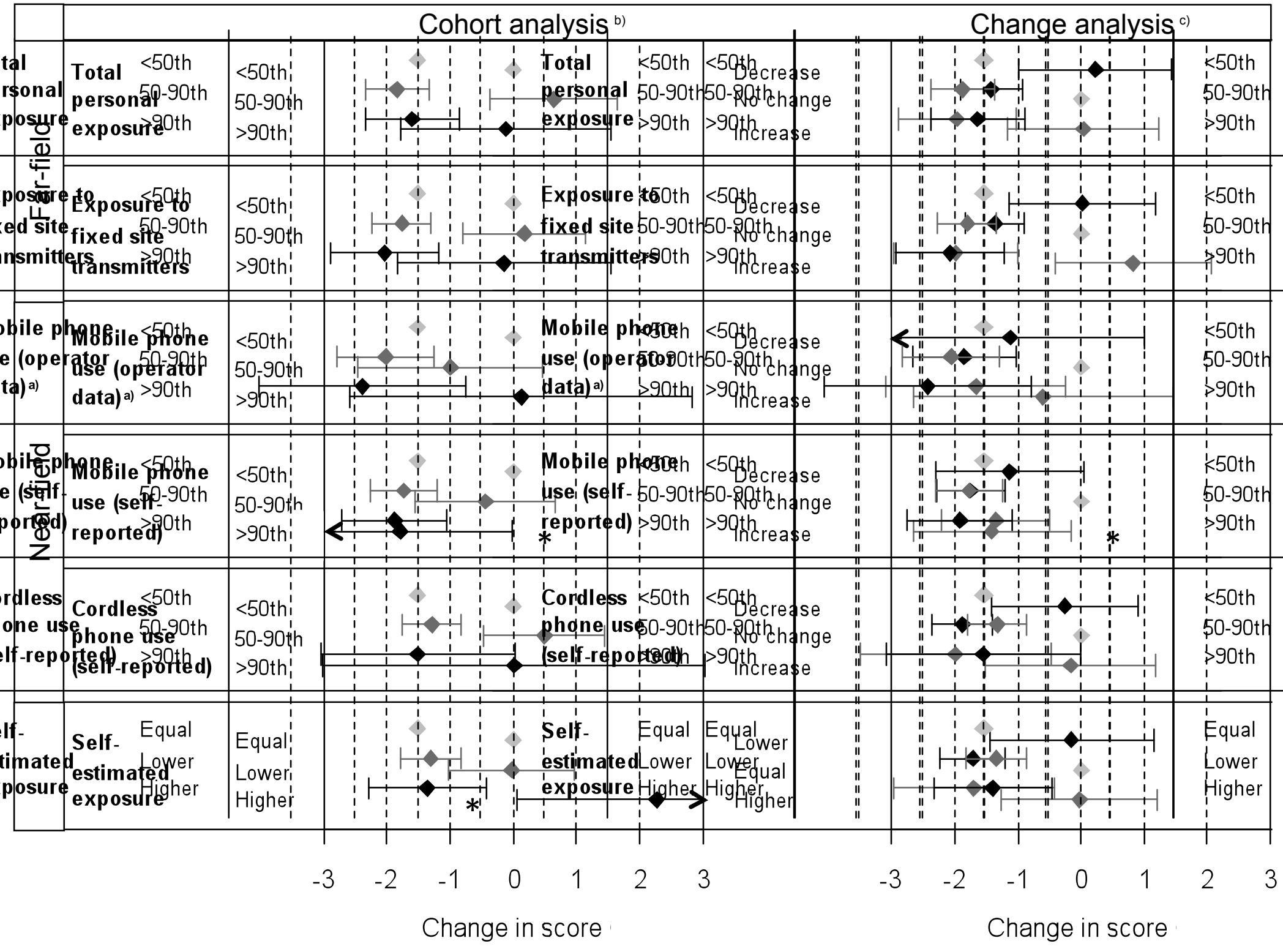


(Figure 2 cont.)

Adjusted for age, sex, body mass index, stress, physical activity, smoking habits, alcohol consumption, education, marital status, degree of urbanity, nightshift work, believe in health effects due to RF-EMF exposure, use of sleeping drugs, general attitude towards the environment and for moving house between the two surveys.

adata from 441 (cohort analysis) and 280 (change analysis) persons

bIn the cohort analysis, the association between exposure at baseline and change in health was investigated cIn the change analysis, the association between change in exposure and health was investigated

${ }^{*} p$-value $<0.05$ (comparison group: individuals exposed below $50^{\text {th }}$ percentile (cohort analysis) and individuals with no (substantial) change of exposure situation (change analysis) 
Figure 3

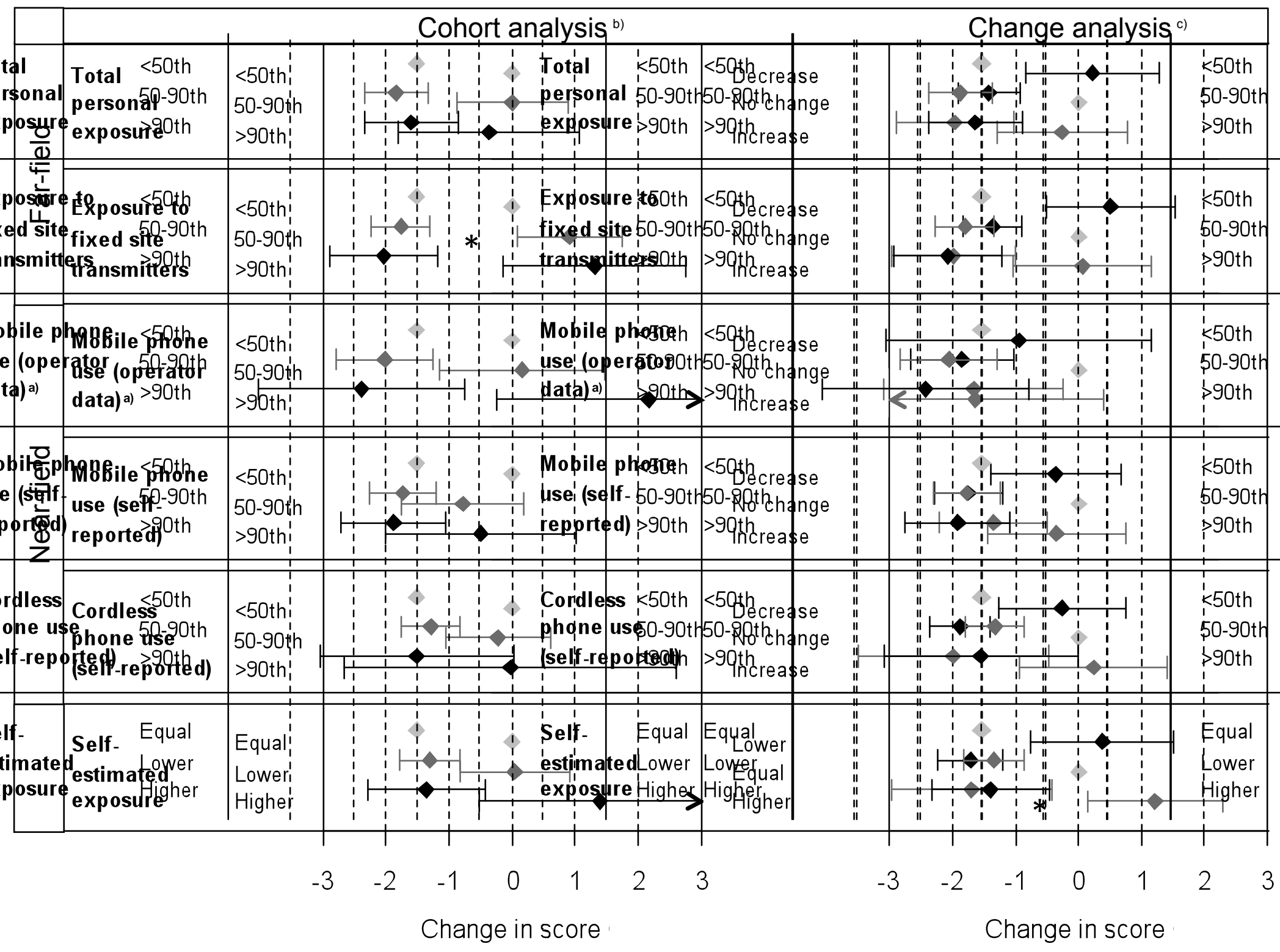




\section{(Figure 3 cont.)}

\section{Confounders see Figure 2}

adata from 451 (cohort analysis) and 284 (change analysis) persons

bln the cohort analysis, the association between exposure at baseline and change in health was investigated CIn the change analysis, the association between change in exposure and health was investigated

${ }^{*}$ p-value $<0.05$ (comparison group: individuals exposed below $50^{\text {th }}$ percentile (cohort analysis) and individuals with no (substantial) change of exposure situation (change analysis) 
Figure 4

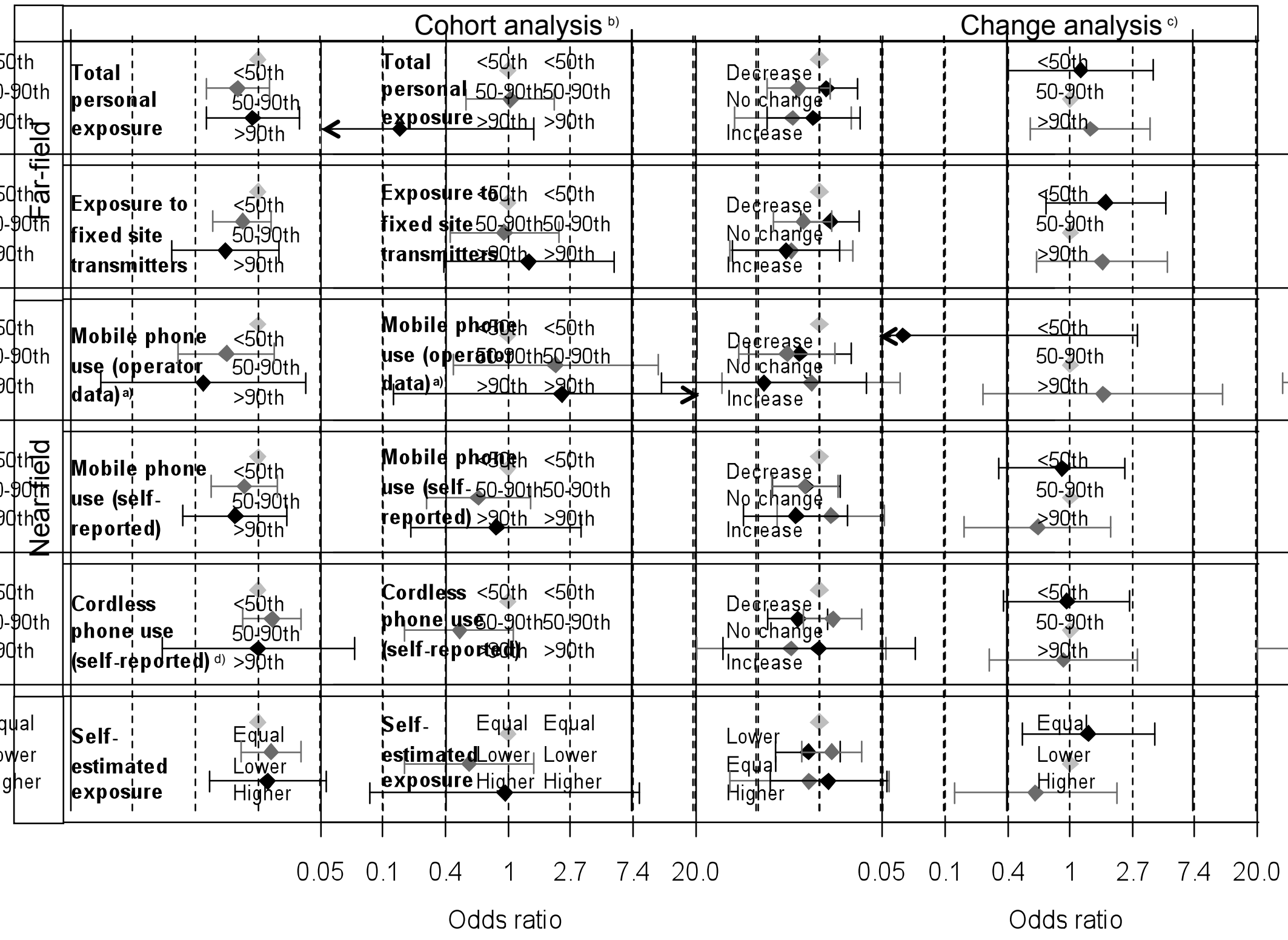




\section{(Figure 4 cont.)}

\section{Confounders see Figure 2}

adata from 455 (cohort analysis) and 286 (change analysis) persons

bIn the cohort analysis, the association between exposure at baseline and change in health was investigated cIn the change analysis, the association between change in exposure and health was investigated

dNo cases observed in the highest exposure group of the cohort analysis

* $\mathrm{p}$-value $<0.05$ (comparison group: individuals exposed below $50^{\text {th }}$ percentile (cohort analysis) and individuals with no (substantial) change of exposure situation (change analysis) 
Click here to download e-components: Appendix Figures A.1-3.pdf 